\title{
Failure mechanisms and electromechanical coupling in semiconducting nanowires
}

\author{
H. D. Espinosa ${ }^{a}$, R. Agrawal, B. Peng ${ }^{\mathrm{b}}$, and R. A. Bernal \\ Northwestern University, Department of Mechanical Engineering, 2145 Sheridan Road, Evanston, \\ IL 60208-3111, USA
}

\begin{abstract}
One dimensional nanostructures, like nanowires and nanotubes, are increasingly being researched for the development of next generation devices like logic gates, transistors, and solar cells. In particular, semiconducting nanowires with a nonsymmetric wurtzitic crystal structure, such as zinc oxide $(\mathrm{ZnO})$ and gallium nitride $(\mathrm{GaN})$, have drawn immense research interests due to their electromechanical coupling. The designing of the future nanowire-based devices requires component-level characterization of individual nanowires. In this paper, we present a unique experimental set-up to characterize the mechanical and electromechanical behaviour of individual nanowires. Using this set-up and complementary atomistic simulations, mechanical properties of $\mathrm{ZnO}$ nanowires and electromechanical properties of $\mathrm{GaN}$ nanowires were investigated. In $\mathrm{ZnO}$ nanowires, elastic modulus was found to depend on nanowire diameter decreasing from $190 \mathrm{GPa}$ to $140 \mathrm{GPa}$ as the wire diameter increased from $5 \mathrm{~nm}$ to $80 \mathrm{~nm}$. Inconsistent failure mechanisms were observed in $\mathrm{ZnO}$ nanowires. Experiments revealed a brittle fracture, whereas simulations using a pairwise potential predicted a phase transformation prior to failure. This inconsistency is addressed in detail from an experimental as well as computational perspective. Lastly, in addition to mechanical properties, preliminary results on the electromechanical properties of gallium nitride nanowires are also reported. Initial investigations reveal that the piezoresistive and piezoelectric behaviour of nanowires is different from bulk gallium nitride.
\end{abstract}

\section{In situ testing of nanostructures}

Semiconducting nanowires (NWs) are potential building blocks for future electronic devices like logic circuits, transistors, and solar cells. NWs made of materials like zinc oxide $(\mathrm{ZnO})$ and gallium nitride $(\mathrm{GaN})$ are interesting for the research community due to their piezoelectric behaviour, offered by the non-symmetric wurtzite crystal structure. For example, ZnO NWs has been conceptually shown to generate electrical output when mechanically deformed [1-3]. To optimize the potential performance of piezoelectric NW-based devices, characterization of individual NWs at the component level is crucial. In this paper, a microelectromechanical system (MEMS)-based

\footnotetext{
${ }^{\mathrm{a}}$ e-mail : espinosa@ northwestern.edu, ${ }^{\mathrm{b}}$ Currently an associate professor in the University of Electronic Science and Technology of China
}

This is an Open Access article distributed under the terms of the Creative Commons Attribution-Noncommercial License 3.0, which permits unrestricted use, distribution, and reproduction in any noncommercial medium, provided the original work is properly cited. 
nanoscale-Material Testing System (n-MTS) to characterize the mechanical and electromechanical behaviour of individual NWs is presented [4].
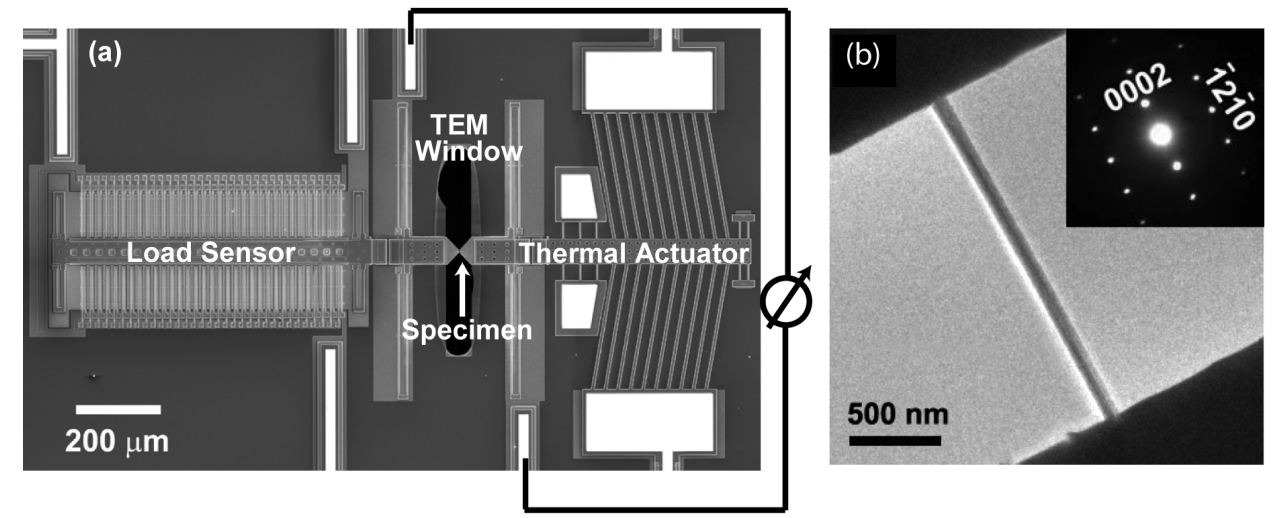

Figure 1 (a) SEM micrograph of a MEMS-based nanoscale electromechanical testing system [4]; (b) a TEM image of a $\mathrm{ZnO}$ nanowire mounted on the testing system [5] (Inset : the diffraction pattern revealing the c-axis orientation of the nanowire)

\subsection{MEMS-based nanoscale-Material Testing System (n-MTS)}

Figure 1a shows the SEM micrograph of the MEMS-based n-MTS, which consists of a thermal actuator and a load sensor [6-8]. A voltage is applied across the thermal actuator beams to achieve displacement controlled loading condition. The applied load is then transmitted to the load sensor through the specimen mounted in between the thermal actuator and load sensor. The displacement of the load sensor is then measured based on a differential capacitive sensing scheme. As the load sensor shuttle is supported on four folded beams, the displacement of the shuttle can be related to the load in the specimen through the stiffness of these folded beams. This set-up can attain nanonewton and nanometer level resolution of loads and displacements respectively. As the load and displacement information is obtained electronically, the n-MTS allows for high resolution imaging of the nanostructure in situ electron microscopes. The specimens are placed on the shuttles of n-MTS using a 3-axis nanomanipulator and fixed via electron beam induced deposition of platinum [8]. One of the main advantages of this experimental set-up is uniaxial tensile/compressive state of loading which makes data interpretation straightforward and facilitates a direct comparison with atomistic simulations with minimal assumptions. This attribute is essential to bridge the gap between existing theoretical and experimental studies [9].

In addition to mechanical testing, the n-MTS also allows for simultaneous electrical measurements [4]. The shuttles on which the specimen is mounted are electrically isolated from rest of the electronic circuitry. Extra traces are laid out so that the specimen can be probed electrically while simultaneously being deformed mechanically. With these features, one can probe the electromechanical coupling in the nanostructures and characterize the piezoresistive and piezoelectric coefficients.

This experimental set-up was used to investigate the mechanical properties of $\mathrm{ZnO}$ nanowires [5, 10] and electromechanical coupling in $\mathrm{GaN}$ nanowires [4]. Computational simulations were performed to understand the experimental findings and to develop predictive capabilities. These results are presented in the next sections.

\section{Mechanical properties of $\mathrm{ZnO}$ nanowires}

$\mathrm{ZnO}$ nanowires, oriented along c-axis with hexagonal cross-section and with diameters ranging from $20 \mathrm{~nm}$ to $400 \mathrm{~nm}$, were mechanically tested using the n-MTS described in previous section [5, 10]. 
The loads were measured from the displacement of the load sensor and stiffness of the folded beams. In order to obtain the displacements for accurate determination of applied strain, two independent but complementary methods were pursued. The first one involved direct imaging of the nanowire specimen to measure its elongation. The second one involved acquisition of nanodiffraction patterns at different stages of loading [5]. From the diffraction patterns, the interatomic spacing was directly calculated to obtain a measure of real atomic level strain. The two methods yielded very similar results which confirmed that there was no slippage occurring at the two welded ends of the nanowire. This validation of fixed-fixed boundary conditions was critical to unambiguously characterize the elastic response of $\mathrm{ZnO}$ nanowires, as earlier studies revealed scattered values for elastic modulus ranging from $20 \mathrm{GPa}$ to $250 \mathrm{GPa}$ with no consensus on the value for a given characteristic size [5, 11-14]. Stress-strain curves for different nanowires were obtained and analyzed in terms of their elastic modulus and fracture strength.

To complement the experimental findings, atomistic simulations were also conducted on nanowires ranging from $1.0 \mathrm{~nm}$ to $20.0 \mathrm{~nm}$ in diameter. Nanowires larger than $5 \mathrm{~nm}$ in diameter were modelled using a semiempirical pairwise Buckingham type potential. The parameters used for this potential were those developed by Binks [15]. The electrostatic interactions were summed following Wolf's method [16], which allows for accurate and computationally efficient modelling of columbic interactions in presence of surfaces [17]. For smaller nanowires, density functional theory (DFT) was used to validate the predictive capabilities of Buckingham potential [18], especially in determining the failure response of the nanowires (as will be highlighted in the Section 2.2). Opensource codes called LAMMPS [19] and SIESTA [20] were used for molecular dynamics simulations and DFT simulations respectively.

\subsection{Elastic properties of $\mathrm{ZnO}$ nanowires}

The elastic response of $\mathrm{ZnO}$ nanowires was found to depend strongly on the characteristic size of the nanowire, particularly for nanowires below $80 \mathrm{~nm}$ in diameter (see Figure 2a). Experiments revealed that the elastic modulus increased from the bulk value of $140 \mathrm{GPa}$ to $160 \mathrm{GPa}$ as the nanowire diameter decreased from $80 \mathrm{~nm}$ to $20 \mathrm{~nm}$ [5]. For diameters larger than $80 \mathrm{~nm}$, elastic modulus close to bulk value of $140 \mathrm{GPa}$ was measured. Similar trend was observed from atomistic simulations using the Buckingham type potential, where the modulus was found to increase from 169 GPa to 192 $\mathrm{GPa}$ as wire diameter decreased from $20 \mathrm{~nm}$ to $5 \mathrm{~nm}$ [5], with $20 \mathrm{~nm}$ size bridging the gap between the experimentally tested nanowire sizes and the computationally modelled ones. The surface effects were analyzed in the computational models to understand the fundamental origin of this size dependence.
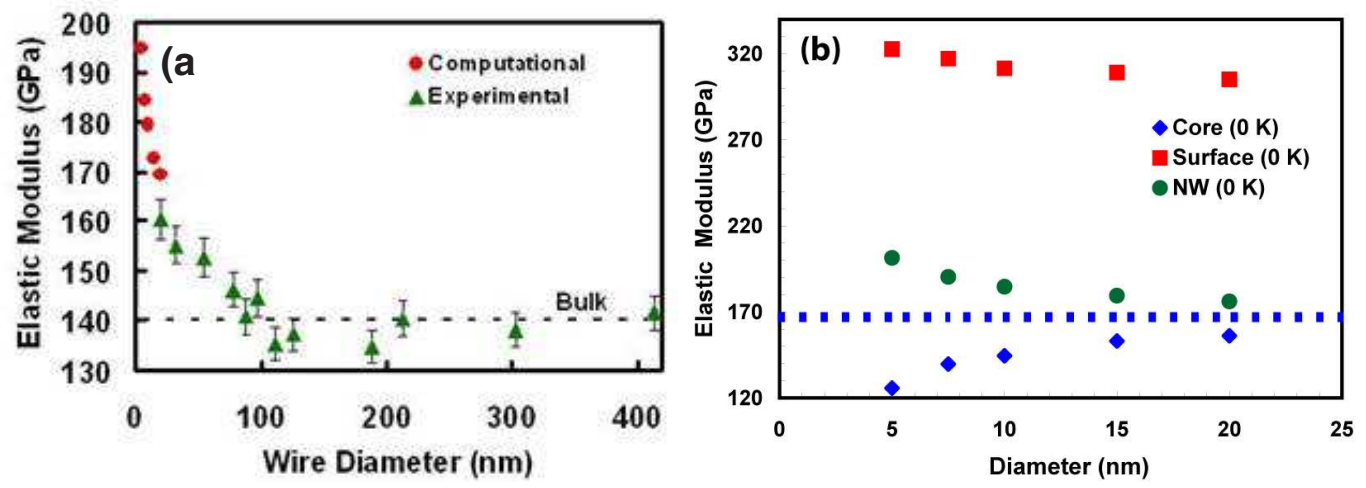
Figure 2 (a) Elastic modulus as a function of nanowire diameter : combined experimental computational results at $300 \mathrm{~K}$ [5]; (b) Elastic modulus contribution from surface and core atoms as estimated from the computations at $0 \mathrm{~K}[5]$.

From the simulations, it was found the surface atoms exhibited a higher stiffness as compared to the bulk (or core) atoms of the nanowire (as shown in Figure 2b). The increased stiffness of the surface atoms was due to reduced interatomic spacing resulting from the surface reconstruction. This effect of surface reconstruction combined with increasing surface to volume ratio as wire diameter decreases lead to the size-dependent trends observed. Using this combined experimentalcomputational approach, we were able to explain the origin of elasticity size effects in $\mathrm{ZnO}$ nanowires [5].

\subsection{Failure mechanisms in $\mathrm{ZnO}$ nanowires}

Even though consistent size dependent results were obtained for the elastic response of $\mathrm{ZnO}$ nanowires from the experiments and the simulations, inconsistent results were obtained for the failure mechanisms. Contrary to experimental reports of brittle fracture $[10,13,21]$ (as shown in Figure 3a), atomistic modelling employing a Buckingham-type pairwise force-field revealed stressinduced phase transformation (shown in Figure $3 b$ ). Simulations showed that the NWs underwent a phase transformation from original wurtzite (WZ) phase to a body-centered tetragonal (BCT) phase $[10,22-23]$. The transformed BCT phase had 4-atom rings of $\mathrm{Zn}$ and $\mathrm{O}$ atoms arranged in a BCTtype structure. The transformation happened at $\sim 6 \%$ strain and the eventual failure of the transformed NW occurs at strains as high as $\sim 18 \%$. This discrepancy between experiments and simulations can be attributed to one of the following three reasons: (i) presence of surface defects in experimentally tested nanowires which were not incorporated in the atomistic models; (ii) inability of the experimental set-up (n-MTS) to be able to capture the deformation path in the loading regime where phase transformation is incipient, and ; (iii) limitation of the pairwise nature of Buckingham type potential in predicting the post-elastic response of these nanowires. Each of these aspects was analyzed in order to understand the reason behind observed differences in experiments and simulations [10].

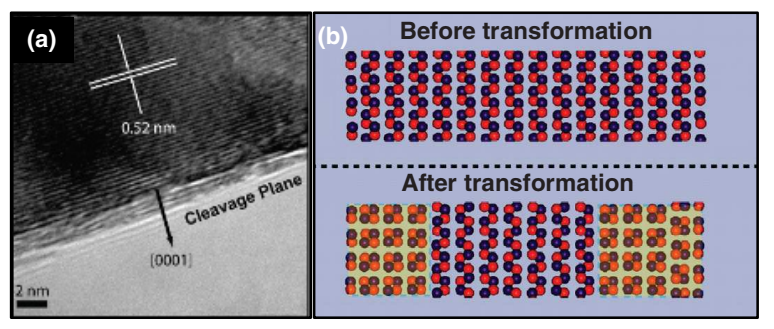

Figure 3(a) Post-mortem TEM image of a $\mathrm{ZnO}$ nanowire revealing brittle fracture along the (0001) cleavage plane [10]; (b) Atomic configurations as predicted by Buckingham type potential before and after the phase transformation for a $10 \mathrm{~nm}$ nanowire [10]. The atoms transformed to BCT phase are shaded for the aid of visulaization.

Firstly, to include the effect of surface defects, the atomistic models with waviness (approximated by sinusoidal functions) were generated [10]. The amplitude of waviness was chosen to be in proportion to the size of surface defects observed experimentally. The nanowire models with surface defects were first annealed to allow for surface reconstructions in presence of surface defects. The stress-strain response was then obtained in a similar fashion as was done for pristine defect-free nanowires. The defects resulted in reduction of critical stresses required for phase transformation; however, the phenomenon of transformation was not completely inhibited. This led 
us to conclude that the phase transformation, if realistic, should occur even in the presence of defects. Secondly, we also realized that the process of phase transformation is accompanied with a dramatic stress relaxation in the stress-strain response. This sudden relaxation leads to an instability in the experimental configuration which makes it extremely challenging to experimentally capture the phase transformation [10].

Lastly, to validate the prediction of pairwise force-fields we are also pursuing first principlesbased DFT calculations to model the stress-strain response of $\mathrm{ZnO}$ nanowires. Due to limitation of computational resources, the number of atoms which can be modelled with DFT is limited. In our preliminary calculations, we modelled a nanowire $1.0 \mathrm{~nm}$ in diameter and two unit cells in length for a comparative study with MM predictions using Buckingham force field. In previous studies, the accuracy of the results obtained with the Buckingham potential was justified by modelling bulk behaviour of WZ and BCT phases using DFT [22-23]. It was reported that the enthalpy of the bulk BCT phase was smaller than that of the bulk WZ phase beyond a critical stress, which established the energetic feasibility of the BCT phase over the WZ phase at high stresses. However, in these studies, the effect of surfaces (as present prominently in the case of nanowires) was never accounted for. We overcome this limitation by modelling actual nanowires with surfaces. In contrast to a phase transformation at $\sim 10 \%$ strain and a secondary loading regime as observed in MM calculations using Buckingham potential, DFT predicts an elastic response up to strains as high as $20 \%$ without any transformation. These findings ascertain that the inherent approximations of the pairwise Buckingham potential can lead to some artefacts in predicting the failure response.

\section{Electromechanical coupling in GaN nanowires}

Using the n-MTS, as described in Section 1, we also investigated the electromechanical properties of GaN nanowires [4]. The electrical response of these nanowires was probed simultaneously while mechanically deforming them to calculate the piezoresistive and piezoelectric coefficients of the nanowires. For accurate characterization of electrical properties in a two-point measurement set-up, measurement of contact resistance resulting from the platinum welds at the two ends of the nanowires is critical. In this protocol, the contact length was systematically varied and the total resistance of the circuit was measured as a function of contact length [4]. This information was then used to extract the contact resistivity at the platinum welds. Preliminary experiments reveal that the electromechanical properties are also influenced by the one-dimensional morphology of nanowires. Piezoresistive and piezoelectric coefficients for nanowires were measured to be higher than their bulk counterpart [4]. Electronic structure calculations, using density functional theory, are being pursued to confirm and complement the experimental findings.

\section{Conclusions}

As evident from the results presented here, very well-defined experiments are required to unambiguously interpret the acquired data with minimal assumptions. This is critical to perform oneto-one comparisons with the computational models. From the experiments, enough atomistic details should be obtained to be incorporated into the models. Similarly, computations should be done on characteristic sizes which can be experimentally tested. Therefore, progress at both the ends i.e. improvisation of in situ experimental techniques with higher resolution and improvement in computational power is the key to be able to bridge the gap between experimentation and theory.

In summary, using combined-experimental approach we were able to resolve the discrepancy concerning elasticity size effects in $\mathrm{ZnO}$ nanowires. Inconsistency in failure mechanisms of these nanowires is revealed and addressed from an experimental as well as computational perspective. In addition to characterization of mechanical properties, we have developed an experimental set-up to probe the electroemechanical behaviour of nanostructures. This type of fundamental studies on individual nanowires and development of predictive capabilities from simulations is essential to formulate design criterion for future nanowires based devices. 


\section{References}

1. Qin, Y., X.D. Wang, and Z.L. Wang, Nature. 451(7180): p. 809-U5.(2008)

2. Wang, Z.L. and J. Song, Science. 312: p. 242.(2006)

3. Yang, R., Y. Qin, C. Li, G. Zhu, and Z.L. Wang, Nano Letters. 9(3): p. 1201-1205.(2009)

4. Peng, B., R. Agrawal, and H.D. Espinosa, Under review in Journal of Micromechanics and Microengineering.(2010)

5. Agrawal, R., B. Peng, E.E. Gdoutos, and H.D. Espinosa, Nano Letters. 8(11): p. 36683674.(2008)

6. Zhu, Y., A. Corigliano, and H.D. Espinosa, Journal of Micromechanics and Microengineering. 16(2): p. 242-253.(2006)

7. Espinosa, H.D., Y. Zhu, and N. Moldovan, Journal of Microelectromechanical Systems. 16(5): p. 1219-1231.(2007)

8. Zhu, Y. and H.D. Espinosa, Proceedings of the National Academy of Sciences of the United States of America. 102(41): p. 14503-14508.(2005)

9. Park, H.S., W. Cai, H.D. Espinosa, and H. Huang, MRS Bulletin. 34(3): p. 178-183.(2009)

10. Agrawal, R., B. Peng, and H.D. Espinosa, Nano Letters. 9(12): p. 4177-4183.(2009)

11. Chen, C.Q., Y. Shi, Y.S. Zhang, J. Zhu, and Y.J. Yan, Physical Review Letters. 96: p. 075505.(2006)

12. Stan, G., C.V. Ciobanu, P.M. Parthangal, and R.F. Cook, Nano Letters. 7(12): p. 36913697.(2007)

13. Desai, A.V. and M.A. Haque, Sensors and Actuators A.(2006)

14. Ni, H. and X. Li, Nanotechnology. 17: p. 3591-3597.(2006)

15. Binks, D.J., Computational Modeling of Zinc Oxide and related oxide ceramics, in Department of Chemistry. 1994, University of Surrey: Harwell.

16. D. Wolf, P. Keblinski, S.R. Phillpot, and J. Eggebrecht, Journal of Chemical Physics. 110: p. 8254.(1999)

17. E. E. Gdoutous, R. Agrawal, and H.D. Espinosa, Under Review in International Journal for Numerical Methods in Engineering.(2010)

18. Agrawal, R., J.T. Paci, and H.D. Espinosa, Manuscript under Preparation.(2010)

19. Plimpton, S.J., Journal of Computational Physics. 117: p. 1-19.(1995)

20. Soler, J.M., E. Artacho, J.D. Gale, A. Garcia, J. Janquera, P. Ordejon, and D. SanchezPortal, Journal of Physics: Condensed Matter. 14: p. 2745-2779.(2001)

21. Hoffmann, S., F. Ostlund, J. Michler, H.J. Fan, M. Zacharias, S.H. Christiansen, and C. Ballif, Nanotechnology. 18: p. 205503.(2007)

22. Wang, J., A.J. Kulkarni, F.J. Ke, Y.L. Bai, and M. Zhou, Computer methods in applied mechanics and engineering. 197: p. 3182-3189.(2008)

23. Wang, J., A.J. Kulkarni, K. Sarasamak, S. Limpijumnong, F.J. Ke, and M. Zhou, Physical Review B. 76(17): p. 172103 (4).(2007) 